\title{
Labyrinthe
}

$8 \mid 2001$

Numéro 8

\section{Les plis de la morale}

Sociologie des fiançailles catholiques

\section{Baptiste Coulmont}

\section{(2) OpenEdition}

Journals

Édition électronique

URL : http://journals.openedition.org/labyrinthe/852

DOI : $10.4000 /$ labyrinthe.852

ISSN : 1950-6031

Éditeur

Hermann

\section{Édition imprimée}

Date de publication : 1 janvier 2001

Pagination : 47-64

\section{Référence électronique}

Baptiste Coulmont, "Les plis de la morale », Labyrinthe [En ligne], 8 | 2001, mis en ligne le 16 mai 2005, consulté le 21 avril 2019. URL : http://journals.openedition.org/labyrinthe/852 ; DOI : 10.4000/

labyrinthe.852

Ce document a été généré automatiquement le 21 avril 2019.

Propriété intellectuelle 


\title{
Les plis de la morale
}

\author{
Sociologie des fiançailles catholiques
}

\section{Baptiste Coulmont}

1 Les années 1930-1940 et le début des années 1990 sont les deux moments du siècle dernier au cours desquels fleurissent les discours sur les fiançailles catholiques. Dans la première moitié du siècle, il s'agissait de créer à la fois un rituel codifié et les usages légitimes de ce rituel : c'est du côté du scoutisme, de la jeunesse ouvrière catholique et des mouvements de familles que s'organisent ces discours. Dans les années 1990, c'est du côté des mouvements charismatiques que se réorganise un nouvel usage rhétorique des fiançailles. Entre ces deux moments, pour diverses raisons tenant entre autres à l'affaiblissement des mouvements catholiques nés dans les années 1920, les fiançailles catholiques disparaissent presque totalement des livres et des revues des années 1960 à la fin des années 1980.

2 Au début des années 1990 sont publiés plusieurs textes sur les fiançailles. Quelques auteurs tentent de remettre au goût du jour les accordailles catholiques. Ces "nouveaux » textes sur les fiançailles sont les œuvres d'hommes appartenant à un cercle d'interconnaissance relativement étroit. Parallèlement à cela, des prêtres fiancent, en sélectionnant les candidats aux fiançailles selon une norme explicite : les bons fiancés sont ceux qui ne vivent pas ensemble. Mettre en regard les injonctions et leurs mises en pratique, (en laissant pour le moment de côté les déclarations des fiancés), montre les réécritures que subissent les entreprises de construction de normes de comportement.

Dans cet article, nous avons tenté d'analyser ces productions sous l'angle de « l'entreprise de morale " au sens de Howard S. Becker. Face à un objet sans contours nets, divers acteurs vont tenter de construire les limites et les usages légitimes de l'objet. D'autres (ou les mêmes) vont mettre en pratique ces normes nouvelles.

4 Les prêtres qui bénissent des fiançailles insistent sur leur originalité en précisant que «c'est mal vu » ou que parfois «le clergé pousse des cris ». Cela reste un signe du peu de légitimité d'un tel geste, mais tous signalent une plus grande sympathie pour les rites à la fois de la part des prêtres et des fidèles. Un aumônier régional de Chrétiens en Grandes 
Écoles, souligne au cours d'un entretien que, depuis dix ans, plusieurs couples lui ont fait part de leur désir de se fiancer, ce qui ne lui était pas arrivé la décennie précédente.

En réponse à mes questions sur ce changement, il déclare que depuis les années 1980 se fait sentir un retour à la ritualisation et qu'est de plus en plus valorisé le côté social et familial du mariage comme cérémonie publique, à l'église (ce qui est un des chevaux de bataille des entrepreneurs de morale dont nous allons parler). Mais ce changement, si changement il y a, ne se remarque pas dans le dépouillement rapide du Journal des Chrétiens en Grandes Écoles (1969-1998), muet sur les fiançailles.

Par quelles voies les fiançailles sont-elles réintroduites dans le "kit » ${ }^{1}$ rituel mis à la disposition des fidèles?

7 «Les normes sont le produit de l'initiative de certains individus, et nous pouvons considérer ceux qui prennent de telles initiatives comme des entrepreneurs de morale ", écrit Howard Becker dans Outsiders (chapitre VIII). « Le prototype du créateur de normes, c'est l'individu qui entreprend une croisade pour la réforme des mœurs [...]. Un tel croisé est fervent et vertueux, souvent même imbu de sa vertu", poursuit le sociologue américain.

Nous nous proposons d'analyser sous cet angle les discours catholiques sur les fiançailles publiés depuis le début des années 1990. Comme nous le verrons, ces textes peuvent être regroupés dans un ensemble à maints égards homogène ; ils reposent sur une rhétorique comparable et sont le support d'une tentative de réforme des mœurs.

Alliance

9 En 1992 paraît un dossier sur les fiançailles dans la revue catholique Alliance. Cette revue, qui tire à 5000 exemplaires et compte 2500 abonnés, est un satellite des Équipes NotreDame, un centre de «spiritualité conjugale " créé par le père Caffarel au sortir de la Seconde guerre mondiale. La revue a été créée en 1979, soit dix ans après les derniers numéros de L'Anneau d'Or, l'organe originel des Équipes Notre-Dame. Elle est dirigée par Michel Rouche, professeur d'histoire à la Sorbonne (Paris IV), compte un «conseiller spirituel» (Xavier de Chalendar), un «conseiller théologique» (un jésuite, Roger Tandonnet) en plus d'un conseil de rédaction. Michel Rouche, cependant, insiste, dans un entretien, sur l'idée qu'Alliance n'est pas une revue catholique ("nous avons une perspective quasiment laïque», me dit-il) et qu'elle n'est pas dirigée par un clerc. L'objectif de la revue est de «diffuser la spiritualité des Équipes Notre-Dame hors des Équipes Notre-Dame» : c'est avant tout une revue reposant sur une rhétorique psychologique. La pensée du professeur Rouche est guidée par les « vérités fondamentales de la psychologie et de la psychanalyse ", vérités fondamentales auxquelles le clergé serait trop souvent aveugle. Ces "vérités » sont les suivantes : les relations sexuelles précoces déclenchent la volonté de plaire à l'autre, pas de le connaître, et sont à l'origine des divorces ; nous vivons une époque de «crise de l'altérité » qui se fourvoie dans «ces bêtises du PACS " (et l'amour homosexuel, qui est un « amour du même», représente le summum de cette crise) ; enfin la "peur de l'engagement », qui qualifie selon Rouche la jeunesse actuelle, lui est révélée dans le refus de la parole publique, qui engage, le dénigrement de tout projet de couple et la trop grande focalisation du couple sur luimême, au mépris des dimensions sociales de l'amour et de l'engagement.

Pour lutter contre toutes ces crises, les fiançailles, telles que les pense Rouche, sont très utiles : elles font reposer le mariage sur un acte d'engagement public et un projet de 
durée qui précède l'union conjugale ; elles sont une période de " connaissance de l'autre » pendant laquelle la possibilité de la rupture doit être constamment présente à l'esprit.

11 Le numéro de 1992 consacré aux fiançailles, intitulé "Un mariage, ça se prépare ", comportait notamment les résultats d'une enquête réalisée par la femme de Michel Rouche, Monique Rouche, auprès de jeunes étudiants parisiens catholiques, plutôt bourgeois, selon le professeur Rouche. Le modèle de cette enquête est l'enquête annuelle menée par les mouvements d'action catholique spécialisée, notamment la JOC, dans les années 1930, qui avait pour but de donner au mouvement les connaissances nécessaires à l'action : aucun résultat chiffré, succession de citations de trois à dix lignes présentant à la fois un visage uni (recherche de la chasteté) et des différences marquées (qui montrent que les chemins individuels sont tous différents). Cette enquête est suivie de la reprise d'un article de L'Anneau d'Or de mai 1960, écrit par « une mère de famille » présentant les fiançailles de sa fille. Michel Rouche embraye ensuite sur une page décrivant «Les fiançailles et l'Église, une histoire conflictuelle », histoire qui s'arrête au XIX siècle. Un article très bien documenté du père Bonnet, supérieur du séminaire d'Issy-LesMoulineaux, examine le droit et la pratique des fiançailles aujourd'hui. Des exemples de cérémonies de fiançailles et de prières des fiancés sont ensuite présentés par Monique Rouche. Enfin, une "thérapeute de couple" présente les bienfaits de la rupture des fiançailles.

12 En 1996, dans un numéro sur "La décision de se marier ", le père Dominique Foyer (prêtre du diocèse de Cambrai, professeur de théologie morale à l'Institut catholique de Lille) propose que l'on sorte du «tout ou rien» en ce qui concerne le sacrement du mariage en revalorisant les fiançailles comme démarche de préparation inaugurée par une « prière en forme de bénédiction » inspirée du Liber Benedictionum.

Hormis le numéro d'Alliance, en 1993, il était difficile à deux jeunes qui souhaitaient se fiancer de trouver un livre sur les fiançailles. Alain Quilici a alors rédigé un livre intitulé Les Fiançailles (aux éditions Le Sarment-Fayard).

Quilici, né en 1936, Toulousain, a terminé son noviciat en 1956, échappe au service militaire en Algérie et s'est - dès son retour à Toulouse - tourné vers la pastorale des jeunes. «Ébranlé » par Vatican II et Mai 1968, il reste néanmoins dans la ligne romaine, est élu à 35 ans prieur du couvent de Marseille, puis Père Maître des novices à Toulouse (1976) pendant quatorze ans. À partir du début des années 1990, il se rapproche de la communauté du Verbe de Vie à Aubazine (fondée en 1986), qui compte selon lui une centaine de membres, filles ou garçons consacrés qui « poursuivent un idéal très proche [de celui des dominicains]». C'est dans ce cadre (participation au Festival des familles...) qu'il rédige Les Fiançailles. Il assume depuis quelques années une chronique mensuelle pour les jeunes dans l'hebdomadaire Famille chrétienne, tout en étant prieur du couvent des dominicains de Toulouse. Il est l'auteur d'une petite dizaine d'ouvrages, dont Les Contes du père Alain Quilici racontés aux petits et aux grands, illustrés par Bruno Rabourdin (connu aussi sous le pseudonyme de Brunor, membre de la communauté de l'Emmanuel depuis 1979, illustrateur travaillant régulièrement pour Famille chrétienne ou pour le magazine pour enfants qui lui est rattaché).

15 Quilici présente donc une figure institutionnelle (il est prieur de Toulouse) proche à la fois des traditionalistes et des charismatiques. Voici comment il me présente son ouvrage, vendu à 8000 exemplaires de 1993 à 1998 :

Mon petit livre ne se veut qu'un guide spirituel pour le temps des fiançailles et de la préparation au mariage ; j'essaye seulement d'aider les jeunes gens qui désirent se 
marier sans mener la vie commune à ne pas perdre leur temps. Mais aujourd'hui, un glissement de sens fait tenir par le plus grand nombre les fiançailles pour une démarche qui précède le mariage mais qui implique la vie commune, avec un accord tacite pour ne pas avoir d'enfants. Souvent, j'entends dire par des jeunes gens qui vivent ensemble parfois depuis plusieurs années qu'ils désirent se fiancer parce qu'ils pensent se marier un jour. Dans leur esprit, ça veut dire qu'ils ne se retiendront plus d'avoir des enfants. (correspondance)

Il présente le « temps des fiançailles » comme un « chemin vers le mariage », chemin qu'il est souhaitable de baliser grâce à un prêtre et à une méditation (une grande partie du livre est constituée d'exemples de méditations à partir de textes bibliques). Le temps des fiançailles est un temps de parole, de prière, de patience et de liberté, un temps de menaces et de dangers (je cite) :

Des fiancés qui n'accepteraient pas très clairement qu'ils sont vraiment libres de se séparer ne se donneraient pas les moyens de mener à bien le temps de leurs fiançailles. (Quilici, 1993, 167)

Alain Quilici consacre enfin quelques pages à la célébration des fiançailles, qui « ne doit pas être une sorte de messe de mariage " (Quilici, 1993, 200) mais bien plutôt un « temps de prière » (et il recommande que, en présence d'un prêtre, une prière soit préférée à une eucharistie).

L'auteur a pour objectif de donner une consistance spirituelle à un mot, un rite et une période qui sont trop flous : "On ne sait plus de quoi on parle ", m'écrit-il. Il remarque que le terme fiançailles est utilisé dans deux contextes différents: soit pour parler de l'état de jeunes qui vivent ensemble et pour qui «le mariage n'est qu'une possibilité parmi d'autres » (Quilici, 1993, 18), soit pour qualifier la «"tradition" » (Quilici utilise les guillemets) de " "certains milieux" » (idem) qui "trouvent de bon ton qu'on se fiance "officiellement" avant de se marier» pour donner un titre aux futurs époux. Le dominicain refuse ces deux emplois pour en proposer un centré sur la non-cohabitation et la spiritualité du mariage.

En 1994, l'hebdomadaire Famille chrétienne propose un dossier de quatre numéros, consacré aux fiançailles catholiques.

Famille chrétienne est un magazine publié par la Société d'Éditions pour la Famille, Édifa, autre nom de la Société d'éditions familiales européennes. Il a été créé en 1977 et fait suite au rapprochement en 1972 de deux sociétés d'éditions, Ecclesia, créée au lendemain de la Seconde Guerre mondiale, et France catholique, issue de la Fédération Nationale Catholique du général de Castelnau. La société Édifa édite aujourd'hui une demi-douzaine de magazines, dont deux pour enfants.

21 En 1994, au moment de la publication du dossier sur les fiançailles, le magazine traditionaliste s'était lancé dans une entreprise de développement, tentant de passer de 2 500 abonnés à 5000 (au moyen d'abonnements groupés, de parrainages, de ventes à la sortie des messes...).

Luc Adrian, né en 1957, est journaliste à Famille chrétienne depuis la fin des années 1980. Il est l'auteur de Dieu de joie, guéris nos frères, un recueil de témoignages sur le charisme de guérison (publié par les éditions Chalet et les éditions de l'Emmanuel), d'un Carnet de route d'un pèlerin, Foi dite en passant, illustré par Bruno Rabourdin, publié aux Presses de la Renaissance (Communauté de l'Emmanuel). Il m'indique que les nombreuses demandes des lecteurs l'ont décidé à composer un dossier sur les fiançailles : d'un côté, des parents voulaient montrer à leurs enfants le sens des fiançailles, souhaitaient que leurs enfants se 
fiancent, et de l'autre, des jeunes ne voyaient pas la signification religieuse du rituel social des fiançailles.

Le lectorat du magazine, qu'il qualifie de "protégé ", a fortement apprécié le dossier. "On a été débordé par le succès et devant le nombre de demandes, on a fait un tiré à part du dossier ». Un grand nombre de lettres et de coups de téléphone, selon Adrian, ont salué cette initiative, et le "courrier des lecteurs" publié garde trace de ces lettres abondantes. Le dossier est publié en février 1994, et, en juillet, $\mathrm{M}^{\mathrm{me}}$ A., de Trappes (l'anonymat est de rigueur dans le courrier des lecteurs) écrit :

Cette lettre pour vous dire merci pour les articles sur les fiançailles. C'est très important de bien s'y préparer. La crise grave par laquelle mon mari et moi passons est le résultat d'une préparation bâclée. [...] Ma nièce, qui va se fiancer, lit assidûment vos articles avec son fiancé. (Famille chrétienne, 860)

Dans une des lettres, une jeune fille de terminale lie ces articles au « combat que nous devons mener pour affirmer nos valeurs » (Famille chrétienne, 848, 62).

Quelles sont donc ces valeurs ? Famille chrétienne propose essentiellement la chasteté et la non-cohabitation au travers d'un long entretien avec le père Sonet, et d'un entretien plus court avec le père Jacques Marin. Le père Sonet (né en 1926), auteur de Réussir notre couple , est animateur de «sessions de la dernière chance " pour des couples sur le point de se séparer. Il délivre une vulgate psychologique et ne présente pas, sur ses livres son état clérical : selon lui, c'est «la COHABITATION qui a rendu caduques les fiançailles » (il souligne, correspondance). L'entretien publié dans Famille chrétienne est soutenu par une rhétorique "psy " : maturité, lucidité, pulsion sexuelle, projet, communiquer... qui reprend plus crûment le thème de la nécessaire connaissance de l'autre avant le mariage :

L'approche physique doit commencer durant les fiançailles : je crois qu'il faut aimer

l'odeur de la peau de l'autre! On ne peut s'engager dans une vie de couple si notre

corps n'est pas attiré par le corps de l'autre. (Famille chrétienne, 839, 52)

Le père Marin, contrepoint spirituel du dossier, a écrit en 1990 Aimer, c'est pardonner. Prêtre de la mission de France, originaire d'une famille d'agriculteurs picards, il est prêtre-ouvrier et membre de la CGT (délégué syndical) jusqu'au milieu des années 1970, moment de sa « deuxième conversion » sous l'influence d'une communauté du renouveau charismatique (1974). Parallèlement à cette reconversion spirituelle et sociale, "les difficultés économiques de son usine le conduisent à arrêter " l'apostolat ouvrier. Il est aujourd'hui conseiller spirituel des Communautés du «Verbe de Vie» et des «Béatitudes».

Enfin, en 1995, les époux Timmel publient Pourquoi nous fiancer?, aux Éditions des Béatitudes.

Les époux Timmel, Philippe (né en 1961) et Catherine (1964), ont alors deux enfants, et ils prêchent des retraites de fiancés au sein de la Communauté des Béatitudes, dont ils font partie. Leur livre, tiré à 8000 exemplaires, s'est vendu à 6100 exemplaires de 1995 au début de 1999, soit un chiffre comparable aux autres productions sur les fiançailles mais largement inférieur aux succès des Éditions des Béatitudes (46 000 exemplaires du livre de Georgette Blaquière, 20000 d'Aimer, c'est pardonner, du père Jacques Marin, qui préface le livre des Timmel). Philippe Timmel me présente de la manière suivante les raisons de l'écriture de leur livre : «[...] Il n'y avait aucun ouvrage catholique [...] sur les fiançailles, et consternés par l'augmentation exponentielle des divorces, nous avons décidé de mettre par écrit le fruit de notre expérience personnelle, aussi bien en tant que couple qu'en tant qu'accompagnateur et prédicateurs de retraites pour couple et fiancés » (correspondance). 
Il présente ensuite les deux "motions» de leur livre : "Trouver ou proposer une "solution" ou du moins un élément de réponse au "pourquoi tant de divorces ?" » et rendre grâce à Dieu pour leur "propre cheminement amoureux ", "à l'heure du PACS et autres dérives du genre ».

Leur livre repose sur une rhétorique alternant la culpabilisation suivie de l'annonce d'une rédemption possible et à portée d'atteinte. Ce style est, selon certains enquêtés, caractéristique des mouvements charismatiques. Deux fiancés, avec qui j'ai eu un entretien, ont peu apprécié l'ouvrage :

Tu connais, Victor ${ }^{2}$, un bouquin sur les fiançailles autre que le Quilici ?

Victor - Ben à part le truc des chachas [charismatiques] [...] Oh, mais

c'est une merde !

Agathe - C'est un scandale, moi, je voulais écrire à La Procure !

Victor - C'est une merde ! c'est niais... euh... c'est niais, euh, à la

limite, même, quand toi t'es pas spécialement catho ou que tu te poses

des questions, tu te dis : « C'est pas pour moi, ils sont complètement

cinglés »... [...] sur la couverture, on voit un petit couple un peu niai

zouille...

Agathe - Qui se donne la main. Moi, j’ai halluciné !

Victor - Et puis il y a tout, il y a la durée du bisou, euh... c'était ridicule ! il y a

même un timing, je ne sais plus! Ils disent que un baiser avec la langue c'est déjà un

acte sexuel!

Agathe - Ahah!

Victor - C'est ça !... remarque, ça dépend où est le baiser !

Cette citation, certes anecdotique, montre que la réception des ouvrages sur les fiançailles, même quand ils s'adressent à des fiancés apparemment conquis, n'est pas exempte d'esprit critique.

De ces quatre tentatives de régénération des fiançailles développées au début des années 1990 , nous pouvons in fine retenir quelques éléments. Tout d'abord que ces entreprises de morale n'ont pas eu un grand impact: 8000 exemplaires pour le livre de Quilici, 6000 exemplaires pour celui des Timmel, moins de 5000 exemplaires pour Famille chrétienne et Alliance.

Sans impact mais pas sans homogénéité : Quilici, les époux Timmel, les pères Marin et Sonet sont tous, à un degré ou à un autre, connus des lecteurs de Famille chrétienne; Michel Rouche et sa revue Alliance, moins traditionalistes, obtiennent aussi les signatures de Sonet et d'autres. La plupart sont relativement jeunes : les Timmel n'ont pas quarante ans, les pères Quilici et Marin à peine soixante (la moyenne d'âge du clergé français est un peu plus élevée). La structuration de leurs discours est comparable: crise de l'engagement, opposition au PACS, à la cohabitation. De même que les Équipes NotreDame, écrit Agnès Walch dans la thèse qu'elle leur a consacrée, reposaient sur l'écoute des demandes des catholiques, de même tous sont conscients que la réforme des mœurs ne se fera pas sans le concours des « consommateurs de morale».

Nous n'avons donc pas affaire aux tenants d'une morale pure, esthétique, mais aux partisans d'une morale pour la pratique. D'où l'usage permanent de "témoignages ", de citations, d'exemples, d'anecdotes qui ont pour but de montrer la morale en acte. Comment passe-t-elle à la pratique ? Les fiançailles se cacheraient-elles dans les plis de la morale? 

recueillent des données sur la préparation au mariage, les mariages, les baptêmes et le nombre d'hosties distribuées chaque semaine...). De plus il n'existe pas de cérémonial propre attaché au mot «fiançailles». Elles peuvent, à l'église, prendre de nombreuses formes. Enfin un certain flou lui-même est attaché au substantif « fiançailles », puisque le temps de la préparation au mariage est parfois qualifié de temps des fiançailles, subtilité qu'il faut pouvoir maitriser quand on s'intéresse à la cérémonie elle-même.

41 Nous espérions, grâce au questionnaire, pouvoir cerner plus précisément la réalité statistique des fiançailles catholiques, déployer la variabilité rituelle des cérémonies, comprendre le lien entre fiançailles et préparation au mariage, et recueillir, par écrit, un texte des prêtres sur leur pratique, mettre à jour des normes de comportement revendiquées. Les résultats du questionnaire, sans être négligeables, ne sont pas tout à fait à la hauteur de nos espérances.

Les réponses écrites ont été accompagnées d'entretiens avec une dizaine de prêtres spécialisés dans les mariages des paroisses concernées.

43 Les entretiens et le questionnaire nous donnent les informations suivantes : on trouve des fiançailles catholiques dans les "paroisses » bourgeoises, mais il n’a pas été possible aux prêtres de me dire si, dans leur paroisse, le nombre de fiançailles avait varié au cours de ces dix dernières années. Cette bénédiction apparaît aux yeux des prêtres comme un rite bourgeois.

44 Cependant les fiançailles catholiques, toutes formes confondues, sont très peu nombreuses : 


\begin{tabular}{|c|c|c|}
\hline \multicolumn{2}{|l|}{ Paroisse Nombre de fiançailles } & Nombre \\
\hline & par an & de mariages \\
\hline Versailles, N.-D. des Armées & 10 & \\
\hline Versailles, Cathédrale St.-Louis & 2 & \\
\hline Paris (5), Saint-Séverin & 10 & 60 (préparations) \\
\hline Neuilly, Saint-Pierre & Entre 5 et 7 & 50 \\
\hline Paris (16), Saint-Honoré d'Eylau & 4 ou 5 & 20 \\
\hline Paris (7), Saint-François Xavier & 2 & \\
\hline Paris (7), Saint-Thomas d'Aquin & 1 (en cinq ans) & \\
\hline Paris (8), La Madeleine & $1(«$ pour ne pas mettre $0 »)$ & 11 \\
\hline Paris (5), St-Jacques du Haut-Pas & Idem & \\
\hline
\end{tabular}




\section{Cohabitation}

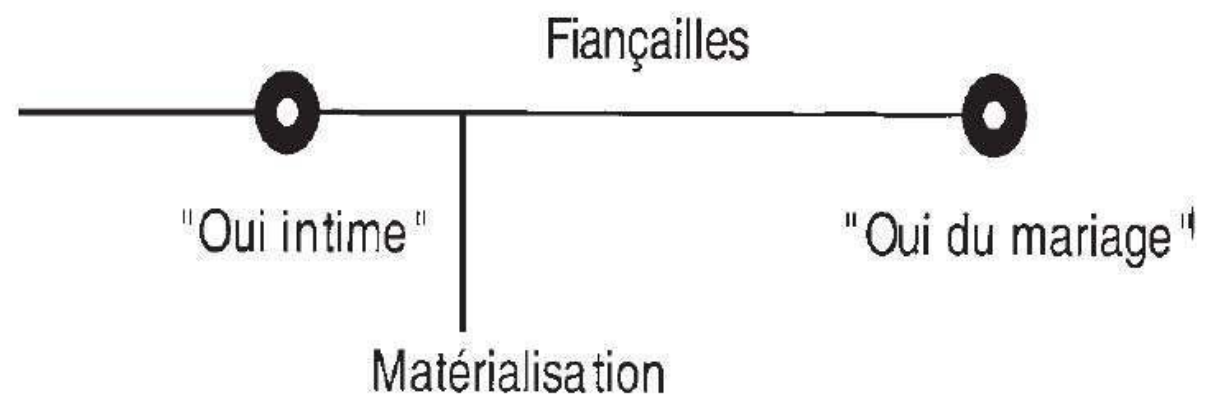

Temps

50 Les « fiançailles » sont ici définies avec audace comme un temps entre un " oui intime, sur l'oreiller » (présenté comme un oui pour la vie) et un «oui du mariage religieux ». On voit, sur ce schéma, qu'elles sont sous-tendues par une cohabitation qui précède à la fois le oui du mariage, mais aussi le oui intime. Dans une conférence prononcée à «L'institut de la famille », le même prêtre écrit :

[...] Les fiançailles sont cette période de la vie où un jeune homme et une jeune femme qui s'aiment vont passer d'un OUI privé à un OUI public, bien plus, d'un OUI individuel à un OUI de couple. (Il souligne)

51 Le document écrit est moins « révolutionnaire », il souligne néanmoins :

Ils (les fiancés) s'aiment et ils sentent combien leur corps est engagé dans cette aventure. Certains ont fait le choix de ne pas avoir de relations sexuelles avant le mariage, d'autres au contraire d'en avoir, tous vont découvrir la force et la pertinence de cette dimension corporelle pour la construction de leur couple.

C'est guidé par cette définition que j'ai construit mon questionnaire, en sachant toutefois combien elle était audacieuse, et ne reflétant en rien une position consensuelle. Les entretiens avec d'autres prêtres n'ont pas plus confirmé cette définition. Mais, à vrai dire, il ne s'est pas dégagé de position bien précise, mis à part le thème du «temps de préparation ».

53 Plusieurs fois même s'est dégagée une position critique : «ça n'a pas vraiment de sens sur le plan religieux » m'a dit un curé, « c'est la matérialisation d'un projet en route, c'est une cérémonie familiale ", « un simili-sacrement », conclut-il. « Ils nous embêtent », m'a dit un vicaire chargé des mariages, parlant des futurs mariés demandant une bénédiction: «Le clergé n'aime pas ça, il est paresseux ». «Au début, j’étais contre, car elles peuvent trop lier les fiancés ", m'a affirmé un autre. Et, même sans position critique sur le sens religieux du rite, tous les prêtres soulignent ce qu'ils nomment la «dimension sociologique » des cérémonies de fiançailles, avant tout des cérémonies bourgeoises, ce qui donne parfois : "Les fiançailles servent à "ferrer l'hameçon" quand les parents de la fille ont trouvé un beau parti.» (vicaire chargé des mariages dans une paroisse bourgeoise de l'Ouest parisien)

54 Comment se déroule la cérémonie des fiançailles ? Mon questionnaire laissait là encore aux prêtres la possibilité de déployer l'éventail des formes. Il ne fut pas grandement 
ouvert, à la fois parce que les fiançailles sont peu nombreuses et parce que les prêtres, individuellement, ont leurs préférences.

Pour comprendre, le plus simple est de relever les oppositions structurant le discours sur le choix cérémoniel. Samedi/dimanche, eucharistie/non, paroisse/extérieur, grand/petit permettent de différencier les fiançailles du mariage et de décrire les bénédictions :

Ce qui est pratique, c'est la messe paroissiale. À la fois pour les familles, car il y a un repas, dans tout ça. Et pour les prêtres : les familles viennent, et ils savent que la messe est dite pour eux. Mais il y en a qui ont un prêtre dans leur poche, des nantis au niveau ecclésiastique, qui demandent la chapelle, en général le samedi aprèsmidi. On n'aime pas trop ça quand ils veulent le faire le dimanche, car le sens ecclésial n'y est pas.

56 On voit ici comment s'opposent le samedi et le dimanche : le samedi, jour "vide », peut être prêté sans problème aux familles, en revanche le dimanche prend ici la valeur opposé de jour " plein », réservé à l'Église.

Ce qui n'est pas méchant, c'est l'intention de prière. Et après la messe, suivant la demande, on lit la bénédiction actuelle (chant d'entrée, texte de l'écriture, un petit mot du prêtre, une intention de prière et une oraison pour les fiancés... la bague, c'est pas à la mode pour le clergé). (le même vicaire)

Les intentions de prière sont apparemment, pour les prêtres, le mode le plus simple d'annoncer des fiançailles religieuses, et surtout, cela n'engage pas autant l'Église qu'une cérémonie à destination spécifique des fiancés, cérémonie présidée par un prêtre: les intentions de prière, en effet, sont dites par des laïcs, ou les fiancés eux-mêmes, ce qui permet aux prêtres de se sentir dégagés de tout investissement.

Mais c'est aussi la bénédiction du pauvre. Comme l'expliquait le vicaire que j’ai déjà plusieurs fois cité, les «nantis » (de capitaux ecclésiastiques) choisissent la messe privée. Un prêtre, conscient de la particularité de «ses» fidèles (des bourgeois parisiens " chrétiens convaincus »), m’a déclaré n'avoir jamais utilisé le moyen de l'intention de prière au cours d'une messe paroissiale. Il fait principalement des messes privées, avec une assistance restreinte ( « c'est arrivé qu'il n'y ait que les deux fiancés, en semaine par exemple »), ou, quand tout le monde manque de temps, une bénédiction après la messe du dimanche.

59 Sur quels critères les prêtres disent-ils se fonder pour accepter de bénir ou non des fiançailles? Elles sont décrites comme affaire de choix familial et de pratique, comme une demande de rite superflu adressée à un prêtre qui se sent donc en droit d'exiger la conformité des demandeurs à la morale théorique du catéchisme.

60 On remarque cela très facilement dans leurs déclarations. D'abord dans le refus, exposé plus haut, de considérer les fiançailles chrétiennes sous l'angle d'une spiritualisation de la cohabitation. Mais aussi dans la description de ce que doivent être de bons fiancés :

Les fiancés qui demandent une cérémonie, ce sont des pratiquants. Pour nous, les prêtres, les fiançailles, c'est pas le mariage, on demande qu'ils restent chastes. S'ils vivent ensemble, je refuse de bénir leurs fiançailles. J'en propose quand ils en remplissent les conditions. (prêtre rattaché à La Madeleine)

61 Ce qui est déclaré compter le plus, donc, c'est l'affichage de la non-cohabitation :

Dans le cadre de la préparation au mariage, nous rencontrons des jeunes qui vivent ensemble 9 fois sur 10, ce qui exclut le concept de fiançailles. S'ils ne vivent pas ensemble, nous pouvons aborder la question des fiançailles et faire une proposition dans ce sens [...] (Versailles, Saint-Louis, correspondance) 
62 dun autre côté, les mêmes prêtres se déclarent conscients de l'efficacité des fiançailles sur le plan familial. Ils savent qu'elles permettent aux fiancés d'entrer dans leur future belle-famille. L'un d'eux m'a dit en conclusion de l'entretien qu'il était à ses yeux « important de fêter les étapes ». Pour cette raison, ils trouvent "intéressant » de célébrer des fiançailles, et cela d'autant plus qu'ils apprécient les explorations rituelles.

À l'apparente homogénéité de la notion de "fiançailles " proposée par les entrepreneurs de morale s'oppose l'apparente incertitude des prêtres paroissiens au sujet des mêmes fiançailles: si les uns ont réussi à construire, combinant les relais éditoriaux traditionalistes et charismatiques, un usage théorique des fiançailles, les autres ont plus de mal à comprendre les tenants et les aboutissants de la cérémonie que de futurs mariés leur demandent.

C'est donc bien dans les plis de la morale, dans la réécriture que subit la morale théorique quand elle passe à la pratique, que se nichent les fiançailles : si théoriquement, c'est l'accès aux relations sexuelles avant le mariage qui pose problème, pratiquement, c'est de la cohabitation qu'il s'agit. Les fiançailles doivent permettre de faire passer de jeunes couples catholiques au mariage religieux. Et la cohabitation, plus que le partage du plaisir sexuel, est perçu comme dangereux pour l'institution.

\section{BIBLIOGRAPHIE}

BECKER Howard S., Outsiders, Paris, Métailié, 1985 (1963).

GRANGe Cyril, Les Gens du Bottin Mondain, y être c'est en être, Paris, Fayard, 1996.

LANEYRIE Philippe, Les Scouts de France, Paris, Cerf, 1985.

Quilici Alain, Les Fiançailles, Paris, Le Sarment-Fayard, 1993.

TIMmel Philippe et Catherine, Pourquoi nous fiancer?, Paris, Éd. des

Béatitudes, 1994.

\section{NOTES}

*. Cet article a été établi à partir d'un mémoire de DEA dirigé par Mme Danièle HervieuLéger (EHESS).

1. J'emprunte cette notion heuristique à Anne-Marie Thiesse, La Création des identités nationales, Paris, Seuil, 1998.

2. Les noms ont été modifiés. 


\section{RÉSUMÉS}

Cet article propose d'analyser deux groupes de discours sur les fiançailles catholiques. D'un côté, plusieurs "entrepreneurs de morale" se rattachant à des mouvements charismatiques ou traditionalistes catholiques tentent de construire un usage codifié des fiançailles. De l'autre, des prêtres paroissiaux confrontés à des demandes de fiançailles, très peu nombreuses mais provenant de jeunes catholiques convaincus, sélectionnent les «bons » candidats aux fiançailles*.

Family bonds

The innermost recesses of morals. Sociology of Catholic betrothals

This article analyses two sets of discourses about Catholic betrothals. On the one hand, a number of " moral entrepreneurs ", who are attached to charismatic or traditionalist Catholic movements, try to follow a codified set of principles for betrothals. On the other hand, some parish priests, facing requests from a small number of strongly Catholic young couples for betrothal ceremonies, attempt to differentiate between « good» and « bad » candidates.

\section{AUTEUR}

\section{BAPTISTE COULMONT}

Baptiste Coulmont est ancien élève de l'École normale supérieure, agrégé de sciences économiques et sociales. Après un DEA sur les tentatives catholiques de construire un rituel des fiançailles, il commence une thèse de sociologie (sous la direction de Danièle Hervieu-Léger, CEIFR-EHESS) portant sur la mise en place de nouveaux rituels de mariage dans les dénominations chrétiennes américaines. Il enseigne actuellement à l'Institute of French Studies de la New York University. Bc4(at)nyu.edu 